\title{
Neoplasias benignas no carcinoides del apéndice cecal: presentación de 36 casos durante los últimos 17 años en un hospital de tercer nivel
}

\author{
Benign non-carcinoid neoplasms of the cecal appendix: presentation of 36 cases during \\ the last 17 years in a third level hospital \\ Jeancarlos J. Trujillo-Díaz ${ }^{*}$, María Ruiz-Soriano", Javier Ortiz de Solórzano-Aurusa1, \\ Beatriz De Andrés-Asenjo', Daniel Corrales-Cruz y Juan P. Beltrán-de Heredia y Rentería ${ }^{1}$ \\ ${ }^{1}$ Servicio de Cirugía General y del Aparato Digestivo; ${ }^{2}$ Servicio de Anatomía Patológica. Hospital Clínico Universitario de Valladolid, Valladolid, España
}

\begin{abstract}
Resumen
Antecedentes: Las tumoraciones del apéndice cecal son un grupo raro y heterogéneo de neoplasias con evolución y pronóstico variables, y tienen una incidencia muy baja. Las tumoraciones mucinosas se definen como aquellas con un apéndice cecal dilatado debido a una acumulación anormal de mucina en su interior; pueden ser benignas o malignas. Dentro de ellas se encuentran los mucoceles, que según los últimos consensos y clasificaciones ya no los incluyen. En este artículo se describe la experiencia de los autores en estas tumoraciones y se propone un grupo en el que incluir los mucoceles; además, se hace una revisión de la literatura. Método: Estudio descriptivo y retrospectivo en el que se analizan todas las piezas quirúrgicas de apéndice cecal durante los últimos 17 años. Resultados: Se analizaron 4910 piezas quirúrgicas. De ellas, 36 fueron incluidas en el grupo de neoplasias epiteliales benignas no carcinoides (NEBNC): 16 fueron neoplasias mucinosas de bajo grado, 14 mucoceles y 6 adenomas tubulares. La forma de presentación más frecuente fue simulando una apendicitis aguda. El tratamiento quirúrgico fue mediante abordaje abierto en 22 casos y laparoscópico en 14. Conclusiones: Las NEBNC sintomáticas pueden simular cuadros de apendicitis aguda, pero en personas con una mayor edad media, por lo que hay que considerar esta posibilidad diagnóstica en pacientes mayores con pruebas de imagen inespecíficas. El tratamiento recomendable es siempre quirúrgico, y resulta viable su realización mediante abordaje laparoscópico.
\end{abstract}

PALABRAS CLAVE: Mucoceles. Neoplasias apendiculares. Neoplasias benignas apendiculares. Neoplasias no carcinoides apendiculares. Tumoraciones mucinosas apendiculares.

\begin{abstract}
Background: The tumors of the cecal appendix are a rare and heterogeneous group of neoplasms with variable prognosis and evolution, its incidence is very low. Mucinous tumors are defined as a dilated cecal appendix due to an abnormal accumulation of mucin, which may be benign or malign. Within these we find the mucoceles, which according to the latest consensus and classifications no longer include them. In this article we describe our experience in these tumors, we also propose a group to include the mucoceles and we review the literature. Method: Descriptive and retrospective study, analyzing all the surgical pieces of cecal appendix during the last 17 years. Results: We analyzed 4910 surgical pieces. 36 were included in the group of non-carcinoid benign epithelial neoplasms (NEBNC), in this group 16 were low-grade mucinous neoplasms, 14 mucoceles and 6 tubular adenomas. The most frequent presentation's form was simulating an acute appendicitis. Surgical treatment was performed by open approach in 22 cases and by laparoscopic approach in 14 cases. Conclusions: Symptomatic
\end{abstract}

\footnotetext{
Correspondencia:

*Jeancarlos J. Trujillo-Díaz

Avda. Ramón y Cajal, 3

C.P. 47003, Valladolid, España

E-mail: jeancar5671@gmail.com
}

Fecha de recepción: 28-09-2018

Fecha de aceptación: 15-12-2018

DOI: $10.24875 / C I R U .18000777$
Cir Cir. 2019;87:630-635 www.cirugiaycirujanos.com 
NEBNC can simulate acute appendicitis but in people with a higher mean age, so this diagnostic should be considered in older patients with nonspecific imaging tests. The recommended treatment is always surgical and it can be possible by laparoscopic approach.

KEY WORDS: Mucoceles. Appendiceal neoplasms. Benign appendiceal neoplasms. Non-carcinoid appendiceal neoplasms. Appendiceal mucinous tumors.

\section{Introducción}

Las tumoraciones del apéndice cecal constituyen un grupo raro y heterogéneo de neoplasias con evolución y pronóstico variables. Tienen una baja incidencia y se estima que corresponden al $0,5 \%$ de todas las neoplasias gastrointestinales'.

Las neoplasias epiteliales no carcinoides (NENC) del apéndice cecal son uno de los subgrupos de tumoraciones apendiculares. Dentro de este subgrupo se encuentran las tumoraciones mucinosas apendiculares, que se definen como aquellas con un apéndice cecal dilatado debido a una acumulación anormal de material mucinoso en su interior, y que pueden ser secundarias a procesos benignos o malignos ${ }^{2}$. Son una afección rara, que corresponde al $0,2-0,7 \%$ del total de las muestras de apendicectomías y representa en torno a un $8-10 \%$ de todas las tumoraciones apendiculares. Suelen ser más frecuentes en las mujeres que en los hombres ${ }^{3}$.

Su diagnóstico suele ser incidental en aproximadamente un $50 \%$ de los casos, durante la realización de pruebas de imagen o endoscópicas, durante la intervención quirúrgica o durante el estudio anatomopatológic $0^{4}$.

El tratamiento recomendable es el quirúrgico, dado que a partir solo de pruebas de imagen no es posible corroborar su benignidad o malignidad 5 .

\section{Método}

Se realizó un estudio descriptivo y retrospectivo en el que se analizaron todas las piezas quirúrgicas de apéndice cecal de los últimos 17 años (enero de 2000 a diciembre de 2017), con el objetivo de identificar la incidencia, las características demográficas, clínicas y radiológicas, el tratamiento quirúrgico realizado, el diagnóstico anatomopatológico y la morbimortalidad en las tumoraciones apendiculares, y además se llevó a cabo una revisión de la literatura.

\section{Resultados}

Se analizaron 4910 piezas de apéndices cecales. De ellas, $36(0.73 \%)$ se incluyeron en el grupo de neoplasias epiteliales benignas no carcinoides (NEBNC) del apéndice cecal; además, 28 fueron diagnosticados como tumores neuroendocrinos $(0.57 \%)$ y 8 como adenocarcinomas (0.16\%). En el grupo de las NEBNC, 16 (44.4\%) fueron neoplasias mucinosas de bajo grado, 14 (38.9\%) fueron mucoceles y $6(16.7 \%)$ fueron adenomas tubulares (Tabla 1 y Fig. 1).

Del total de las NEBNC, 22 (61.1\%) correspondieron a mujeres y $14(38.9 \%)$ a hombres. La edad media de presentación fue de 67 años. La longitud media de los apéndices fue de $7.15 \mathrm{~cm}(3-13 \mathrm{~cm})$ y el diámetro medio fue de $2.5 \mathrm{~cm}(0.6-5.5 \mathrm{~cm})$. La estancia hospitalaria media fue de 7.9 días (1-28 días). En el seguimiento no se registró ninguna muerte (Tabla 2 ).

La forma de presentación más frecuente fue simulando una apendicitis aguda, en 18 casos (50\%), seguida por dolor abdominal crónico en 7 casos (19.4\%), hallazgo incidental en cirugía de cáncer de colon en 6 casos (16.7\%), hallazgo en colonoscopia de cribado en 2 casos (5.6\%) fueron, masa abdominal palpable en 1 caso (7.1\%), invaginación ileocecal en un caso y oclusión intestinal en un caso. En 15 piezas quirúrgicas del grupo de NEBNC (41.7\%) se encontraron signos histológicos de apendicitis aguda (Tabla 3).

El tratamiento quirúrgico fue mediante abordaje abierto en 22 casos (61.1\%) y laparoscópico en 14 (38.9\%). El tipo de intervención quirúrgica fue solo apendicectomía en 18 casos (50\%), apendicectomía más resección cecal en 8 casos $(22.2 \%)$ y hemicolectomía derecha en 7 casos (19.4\%) (Tabla 4).

\section{Discusión}

Uno de los términos inicialmente utilizados para describir las tumoraciones mucinosas apendiculares fue la de «mucocele». El término «mucocele apendicular» fue definido por primera vez en 1842 por Karel Rokitansky como la dilatación del apéndice cecal con acumulación de material mucinoso en su interior ${ }^{3}$. Esto incluye una amplia variedad de tumores, que comprenden desde quistes simples de retención hasta adenocarcinomas invasivos, pero actualmente ha quedado obsoleto ${ }^{6}$. 
Tabla 1. Subtipos histológicos encontrados en las piezas quirúrgicas

\begin{tabular}{lcc}
\hline & $\mathbf{n}$ & $\%$ \\
\hline Total de apendicectomías (2000-2017) & 4910 & \\
Neoplasias benignas no carcinoides & 36 & $0,7 \%$ \\
Lesiones epiteliales & 22 & $61,1 \%$ \\
Adenoma tubular & 6 & $16,7 \%$ \\
Neoplasia mucinosa de bajo grado & 16 & $44,4 \%$ \\
Mucoceles & 14 & $38,9 \%$ \\
\hline
\end{tabular}

Tabla 2. Características demográficas de los pacientes en estudio

\begin{tabular}{lcc}
\hline & $\mathbf{n}$ & $\%$ \\
\hline GÉNERO: Mujeres & 22 & $61,1 \%$ \\
Hombres & 14 & $38,9 \%$ \\
Edad media (años) & 67 & $37-87$ años \\
Tamaño apendicular medio longitud (cm) & 7.15 & $3-13 \mathrm{~cm}$ \\
Diámetro (cm) & 2.5 & $0,6-5,5 \mathrm{~cm}$ \\
Estancia hospitalaria (días) & 7,9 & $1-28$ días \\
\hline
\end{tabular}

Tabla 3. Formas de presentación de las neoplasias benignas no carcinoides

\begin{tabular}{lcc}
\hline Formas de presentación & $\mathbf{n}$ & $\%$ \\
\hline Apendicitis aguda & 18 & $50 \%$ \\
Dolor abdominal crónico & 7 & $19,4 \%$ \\
Cáncer de colon & 6 & $16,7 \%$ \\
Colonoscopia de cribado & 2 & $5,6 \%$ \\
Masa abdominal palpable & 1 & $2,8 \%$ \\
Invaginación íleo-cecal & 1 & $2,8 \%$ \\
Oclusión intestinal & 1 & $2,8 \%$ \\
\hline
\end{tabular}

Tabla 4. Tipos de abordajes y de cirugías realizadas

\begin{tabular}{lcc}
\hline & $\mathbf{n}$ & $\%$ \\
\hline Abordaje Abierto & 22 & $61,1 \%$ \\
Laparoscópico & 14 & $38,9 \%$ \\
Tipo de cirugía Apendicectomía & 18 & $50,0 \%$ \\
Apendicectomía+resección cecal & 8 & $22,2 \%$ \\
Hemicolectomía derecha & 7 & $19,4 \%$ \\
Otros & 3 & $8,4 \%$ \\
\hline
\end{tabular}

Actualmente, la clasificación de las NENC del apéndice cecal, dentro de la cual se encuentran las tumoraciones mucinosas, es controversial, e incluso llega a ser confusa, debido a que no hay un consenso establecido en los términos utilizados ${ }^{7}$.

La clasificación clásica las subdividía en mucocele simple o quiste de retención, hiperplasia de la mucosa, cistoadenoma mucinoso y cistoadenocarcinoma mucinoso ${ }^{8}$.

Misdraji, et al., ${ }^{910}$ las clasificaron en tumores mucinosos de bajo grado (ausencia de infiltración, confinados al apéndice o con diseminación al peritoneo) y en adenocarcinomas (presencia de infiltración). Posteriormente, Pai y Longacre ${ }^{11}$ añadieron dos nuevas categorías: neoplasia mucinosa de potencial incierto maligno y neoplasia mucinosa de bajo potencial maligno.

Malya, et al. ${ }^{2}$ utilizan en su trabajo una terminología similar a la de Misdraji, et al.9: tumores mucinosos apendiculares de bajo grado y adenocarcinoma; sin embargo, dentro del grupo de tumores mucinosos de bajo grado incluyen el mucocele simple o quiste de retención, la hiperplasia de la mucosa y el adenoma mucinoso, catalogándolos como tumoraciones mucinosas benignas del apéndice.

Según la clasificación de la Organización Mundial de la Salud, las NENC del apéndice cecal abarcan el adenoma, la neoplasia mucinosa de bajo grado, el adenocarcinoma mucinoso, el carcinoma de células en anillo de sello y el adenocarcinoma no mucinoso².

Según el Peritoneal Surface Oncology Group International (PSOGI) y el último consenso del American Joint Committee on Cancer (AJCC), la terminología que debería utilizarse sería la de neoplasias no invasivas (adenoma, pólipo serrado con o sin displasia, neoplasia mucinosa apendicular de bajo y alto grado) y neoplasias invasivas (adenocarcinoma mucinoso, carcinoma de células en anillo de sello y adenocarcinoma no mucinoso) $)^{3}$.

Como puede verse, no hay unanimidad en la clasificación y en los términos utilizados, y existe mucha controversia acerca de qué clasificación debería utilizarse. En las últimas clasificaciones ya no se incluye lo que se conocía clásicamente como mucoceles simples. Nosotros nos hemos basado en la clasificación utilizada por Malya, et al. ${ }^{2}$, dado que es la única que incluye los mucoceles simples, y además, utilizando la última clasificación del PSOGI y el AJCC, hemos incluido los mucoceles dentro del grupo de neoplasias no invasivas y las hemos denominado «neoplasias epiteliales benignas no carcinoides del apéndice cecal» (NEBNC) (Fig. 2). 

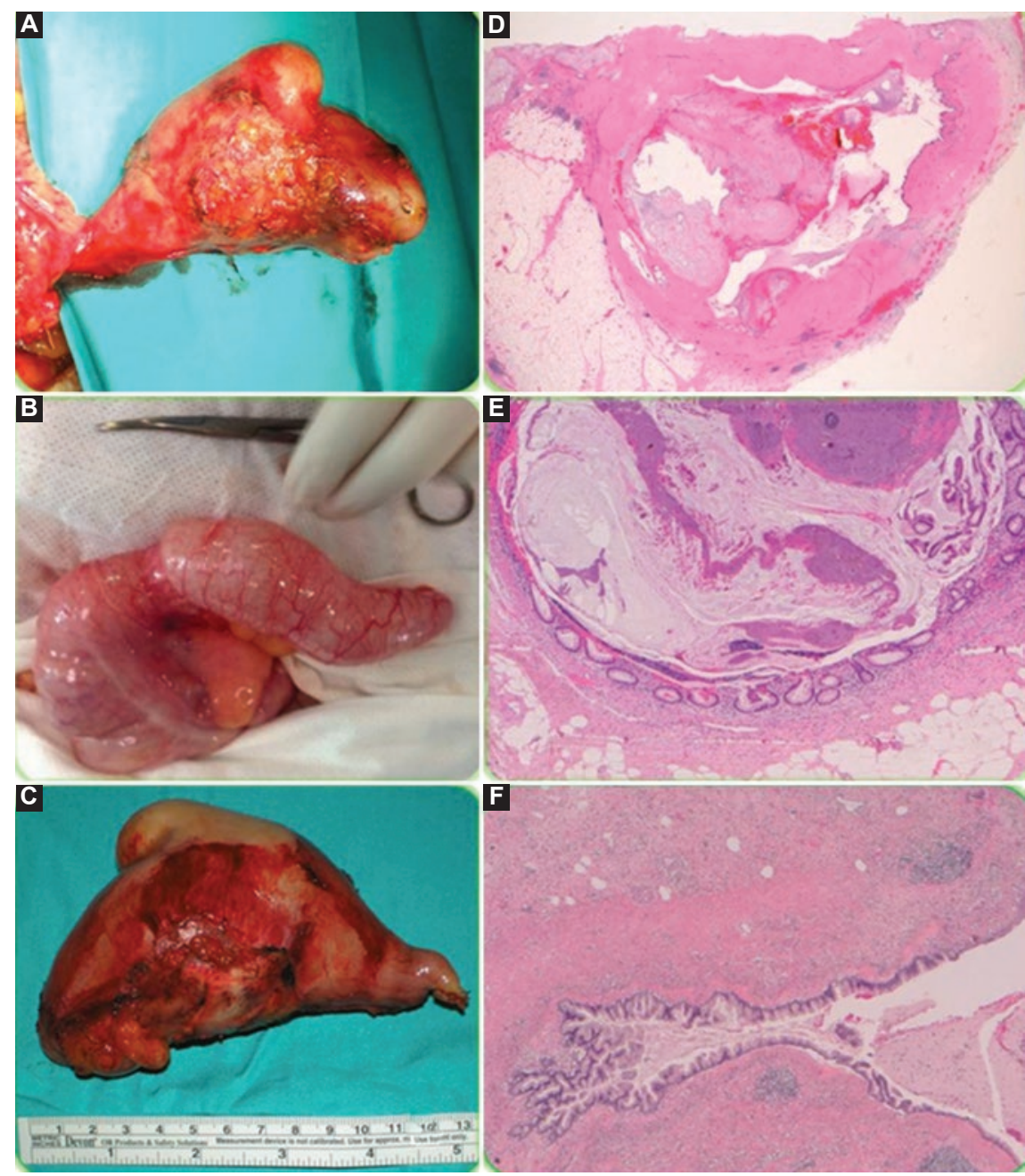

Figura 1. Pieza quirúrgica; A-C: apéndice cecal aumentado de tamaño con inflamación del mesoapéndice. Histología; D: neoplasia mucinosa de bajo grado; E: mucocele; F: adenoma tubular.

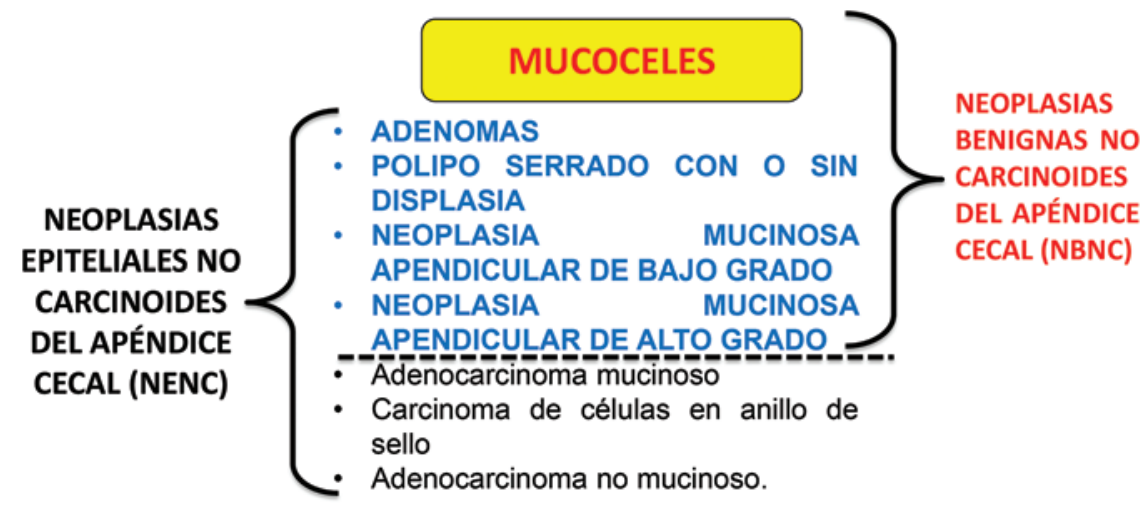

Figura 2. Clasificación propuesta incluyendo al grupo de los mucoceles.

En cuanto a las manifestaciones clínicas, podemos decir que aproximadamente un $50 \%$ de los casos son asintomáticos y su hallazgo es incidental $^{12}$; en los sintomáticos, la molestia más frecuente es el dolor abdominal, que se presenta en más del $80 \%$ de los casos, simulando en su mayoría una apendicitis aguda, como en nuestra serie, en la que el $50 \%$ fueron simulando este cuadro. Otros síntomas son una masa palpable en la fosa ilíaca derecha, náuseas, vómitos, pérdida de peso, 


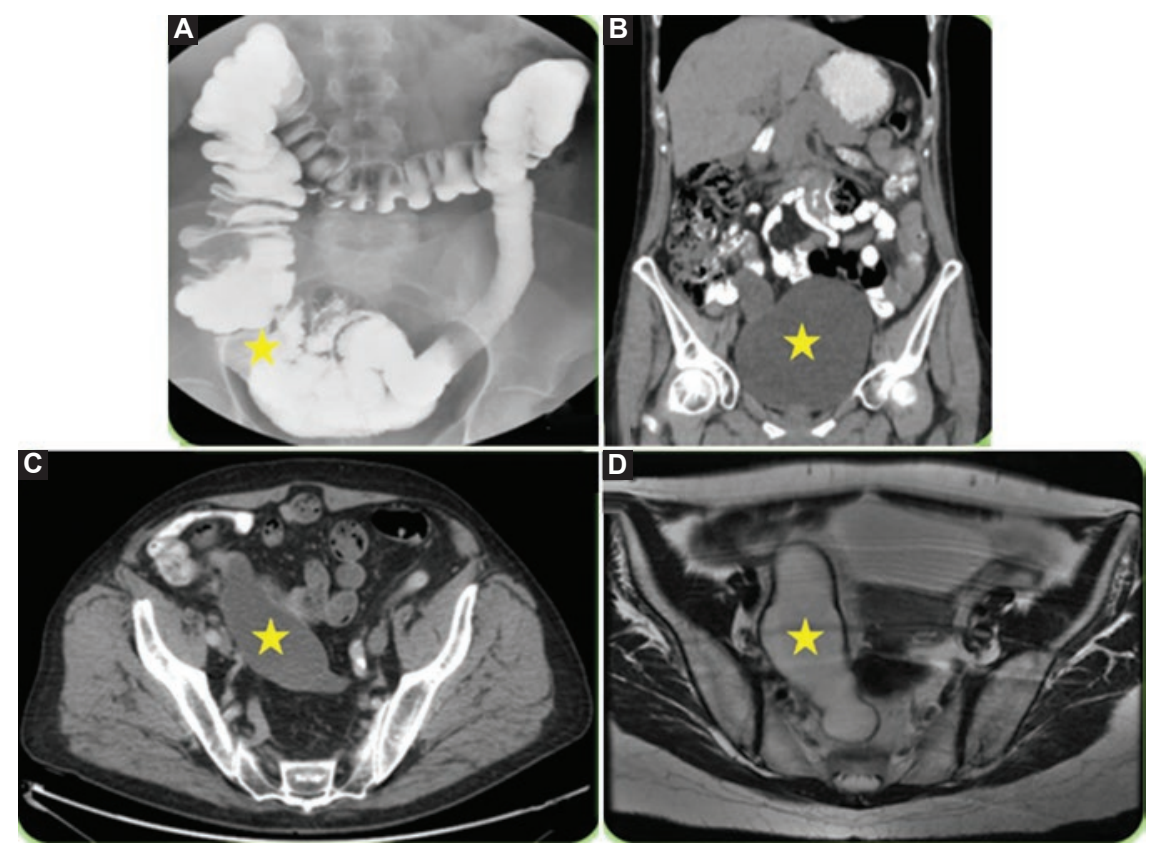

Figura 3. Pruebas de imagen. A: enema baritado; B: corte coronal de tomografía computarizada; C: corte axial de tomografía computarizada; D: resonancia magnética. En todas se visualizan tumoraciones apendiculares aumentadas de tamaño (estrellas) compatibles con neoplasias benignas no carcinoides apendiculares.

obstrucción intestinal y rotura espontánea con peritonitis generalizada 4 .

El diagnóstico de sospecha preoperatorio es un factor importante, dado que orienta a determinar el tipo y la extensión de la cirugía que debe realizarse, pero en general esto es difícil debido a que en un gran número de pacientes el diagnóstico se realiza intraoperatoriamente 0 más tarde durante el estudio anatomopatológico ${ }^{3}$, como ocurrió en la mayoría de los casos de nuestra serie.

Con el uso de técnicas de imagen puede ser posible lograr un diagnóstico preoperatorio correcto hasta en el $30 \%$ de los casos. Las más utilizadas son la ecografía, la tomografía computarizada y la resonancia magnética, las cuales son útiles para evaluar el tamaño, la extensión y las características morfológicas de la lesión² (Fig. 3).

En la ecografía puede observarse una lesión quística encapsulada asociada al ciego y a menudo con calcificaciones en su pared33. Múltiples capas ecogénicas le dan una apariencia en piel de cebolla, que es patognomónica ${ }^{13}$. La tomografía computarizada es la técnica ideal, aunque resulta diagnóstica en menos del $50 \%$ de los casos. Ayuda a distinguir las masas quísticas del apéndice de otras como las ováricas, y además puede ayudar a diferenciar los posibles procesos benignos de los malignos, pues los malignos por lo general son más grandes $(>2 \mathrm{~cm})$, con calcificaciones murales curvilíneas, irregularidades en la pared y alteraciones de la grasa circundante ${ }^{5}$.

Se ha observado una asociación entre las NENC del apéndice cecal y otras neoplasias, entre ellas la colorrectal, por lo que se recomienda realizar una colonoscopia a todos los pacientes en quienes se sospeche su existencia ${ }^{14}$. En nuestra serie, las NEB$\mathrm{NC}$ fueron un hallazgo incidental durante una colonoscopia de cribado en dos pacientes, y en seis con adenocarcinoma de colon.

El diámetro máximo apendicular de las NEBNC suele $\mathrm{ser}<2 \mathrm{~cm}$, por lo que ante diámetros mayores hay que sospechar malignidad. En cuanto a su asociación con la apendicitis aguda, podemos decir que, en ausencia de un apendicolito, el diámetro apendicular no debe ser $>1.5 \mathrm{~cm}$ para un diagnóstico de apendicitis aguda; en caso contrario, habría que pensar que nos encontramos ante una posible NENC ${ }^{2}$.

El manejo terapéutico es siempre quirúrgico, y va desde la apendicectomía hasta una hemicolectomía derecha. En lo que respecta al tipo de abordaje óptimo, sigue siendo controversial. El abordaje laparoscópico es recomendado por algunos autores en pacientes seleccionados, y siempre se realiza con una técnica meticulosa para minimizar el riesgo de rotura iatrogénica y de siembra peritoneal, evitando así el riesgo de aparición de un pseudomixoma peritoneal. Por el contrario, el abordaje abierto 
tiene la ventaja de que permite una manipulación quirúrgica segura de la lesión, evitando así su rotura $^{15}$. Nosotros hemos realizado un abordaje laparoscópico en el 38,9\% de nuestros pacientes, sin que hayan presentado ninguna complicación asociada a corto ni largo plazo.

En los casos de NEBNC, la cirugía debe ser una apendicectomía con resección en bloque de la grasa mesentérica apendicular ${ }^{4}$. Dentro del mesenterio apendicular se encuentran entre cuatro y ocho ganglios linfáticos que, si en el estudio histológico son negativos, una cirugía de extensión como la hemicolectomía derecha no está indicada. Por otro lado, si se encuentra un margen positivo en la base apendicular debe realizarse una cirugía de extensión, que puede ser una resección cecal y así conservar el colon ascendente y la función de la válvula ileocecal ${ }^{15}$. Aquellos casos en los que el apéndice cecal se encuentre perforado, pero con ganglios linfáticos y márgenes negativos, no deben someterse a una hemicolectomía derecha, ya que presentan tasas de supervivencia más bajas en comparación con los que solo se someten a una apendicectomía ${ }^{16}$.

Si el estudio histológico indica carcinomatosis peritoneal mucinosa, se requerirá cirugía citorreductora o quimioterapia intraperitoneal hipertérmica (HIPEC), o ambas, que aumentan la supervivencia a largo pla$\mathrm{zo}^{17}$. La quimioterapia sistémica antes de la cirugía citorreductora o la HIPEC puede mejorar el pronóstico en estos pacientes ${ }^{18}$.

\section{Conclusión}

Las NEBNC sintomáticas pueden simular cuadros de apendicitis aguda, pero su presentación es más frecuente en pacientes con una edad media mucho mayor que en los casos típicos de apendicitis aguda, por lo que hay que considerar esta posibilidad diagnóstica en los pacientes mayores, con pruebas de imagen inespecíficas y con un diámetro apendicular $>2 \mathrm{~cm}$. El tratamiento recomendable es siempre quirúrgico, desde la apendicectomía hasta la hemicolectomía derecha, y su realización es viable mediante abordaje laparoscópico.

\section{Conflicto de intereses}

Los autores declaran que no existe conflicto de intereses en relación con el artículo que se ha remitido para publicación.

\section{Financiamiento}

Los autores declaran que el presente trabajo no ha requerido financiamiento.

\section{Responsabilidades éticas}

Protección de personas y animales. Los autores declaran que para esta investigación no se han realizado experimentos en seres humanos ni en animales.

Confidencialidad de los datos. Los autores declaran que han seguido los protocolos de su centro de trabajo sobre la publicación de datos de pacientes.

Derecho a la privacidad y consentimiento informado. Los autores han obtenido el consentimiento informado de los pacientes y/o sujetos referidos en el artículo. Este documento obra en poder del autor de correspondencia.

\section{Bibliografía}

1. Butte B, García M, Torres J, Salinas M, Duarte I, Pinedo G, et al. Tumores del apéndice cecal: análisis anatomoclínico y evaluación de la sobrevida alejada. Rev Chil Cir. 2007;59:217-22.

2. Malya F, Hasbahceci M, Serter A, Cipe G, Karatepe O, Kocakoc E, et al. Appendiceal mucocele: clinical and imaging features of 14 cases. Chirurgia (Bucur). 2014;109:788-93.

3. Emre A, Sertkaya M, Kale I. Clinicopathological analysis of appendiceal mucinous tumors: a single-center experience. Turk J Surg. 2017;33:274-8.

4. Rymer B, Forsythe R, Husada G. Mucocoele and mucinous tumours of the appendix: a review of the literature. Int J Surg. 2015;18:132-5.

5. Orcutt S, Anaya D, Malafa M. Minimally invasive appendectomy for resection of appendiceal mucocele: case series and review of the literature. Int J Surg Case Rep. 2017;37:13-6.

6. Nutu O, Marcacuzco A, Manrique A, Justo I, Calvo J, García M, et al Mucinous appendiceal neoplasms: incidence, diagnosis and surgical treatment. Cir Esp. 2017;95:321-7.

7. Darriba M, Madrazo Z, Aranda H, Sanjuan X, Hernández J. Neoplasias mucinosas del apéndice: ¿hablamos todos el mismo idioma? Rev Esp Enferm Dig. 2012;104:44-5.

8. Carr N, Bibeau F, Bradley R, Dartigues P, Feakins R, Geisinger K, et al. The histopathological classification, diagnosis and differential diagnosis of mucinous appendiceal neoplasms, appendiceal adenocarcinomas and pseudomyxoma peritonei. Histopathology. 2017;71:847-58.

9. Misdraji J, Yantiss R, Graeme-Cook F, Balis J, Young R. Appendiceal mucinous neoplasm. A clinicopathologic analysis of 107 cases. Am J Surg Pathol. 2003;27:1089-103.

10. Misdraji J. Appendiceal mucinous neoplasm: controversial issues. Arch Pathol Lab Med. 2010;134:864-70.

11. Pai R, Longacre T. Appendiceal mucinous tumors and pseudomixoma peritonei. Adv Anat Pathol. 2005;12:291-311.

12. Kelemouridou E, Mogrampi S, Tsavis G, Verroiotou M, Rallis T, Fardellas I. Mucinous cystadenoma of the appendix. A diagnostic dilemma? Chirurgia (Bucur). 2011;106:251-4.

13. Caspi B, Cassif E, Auslender R, Herman A, Hagay Z, Appelman Z. The onion skin sign: a specific sonographic marker of appendiceal mucocele. J Ultrasound Med. 2004;23:117-23.

14. Abuoğlu H, Yıldız M, Kaya B, Odabaşı M. Clinicopathological analysis of patients operated for appendiceal mucocele. Ulus Travma Acil Cerrahi Derg. 2017;23:230-4.

15. Hamilton D, Stormont J. The volcano sign of appendiceal mucocele. Gastrointest Endosc. 1989:35:453-6.

16. González-Moreno S, Sugarbaker P. Right hemicolectomy does not confer a survival advantage in patients with mucinous carcinoma of the appendix and peritoneal seeding. Br J Surg. 2004;91:304-11.

17. Mohamed F, Cecil T, Moran B, Sugarbaker P. A new standard of care for the management of peritoneal surface malignancy. Curr Oncol. 2011;18:e84-96.

18. Milovanov V, Sardi A, Ledakis P, Aydin N, Nieroda C, Sittig M, et al. Systemic chemotherapy (SC) before cytoreductive surgery and hyperthermic intraperitoneal chemotherapy (CRS/HIPEC) in patients with peritoneal mucinous carcinomatosis of appendiceal origin (PMCA). Eur J Surg Oncol. 2015;41:707-12. 